\title{
Determining the role of TIR domain of Interleukin-1 receptor 8 (SIGIRR) in regulating TLR4 signalling
}

\author{
Surekha Nimma ${ }^{1}$, Jeffrey Nanson ${ }^{1}$, Thomas Ve ${ }^{1,2}$ and Bostjan Kobe ${ }^{1,3}$ \\ ${ }^{I}$ School of Chemistry and Molecular Biosciences and Australian Infectious Diseases Research Centre, University of Queensland, \\ Brisbane, QLD 4072, Australia, \\ ${ }^{2}$ Institute for Glycomics, Griffith University, Southport, QLD 4222, Australia, \\ ${ }^{3}$ Institute of Molecular Bioscience, University of Queensland, Brisbane, QLD 4072, Australia
}

s.nimma@uq.edu.au

The TIR superfamily includes membrane receptors, Interleukin-1 receptors (IL-1Rs) and Toll-like receptors (TLRs) and also TIRcontaining cytoplasmic adaptor proteins such as MAL and MyD88. These proteins play a major role in immune signalling and are vital to innate host defense, inflammation, injury and stress [1]. IL-1R8, also known as single immunoglobulin interleukin-1 receptor-related protein (SIGIRR) is an inhibitory receptor from IL-1R family which regulates signalling of both IL-1Rs and TLRs. The mechanism of inhibition is not yet known, but the only available genetic evidence suggests that the conserved intracellular TIR domain of IL-1R8 alone is necessary to inhibit LPS-induced TLR4 signalling [2]. The recent cryo-EM structure of the MAL protofilament has revealed the molecular mechanism of TIR-TIR interactions in the MAL and MyD88 dependent TLR4 signalling [3]. Based on this, we hypothesize that a similar TIR:TIR interaction between the TIR domain of IL-1R8 and the TIR domains of either TLR4/MAL/MyD88 would be involved in the inhibition mechanism.

The TIR domain of human IL-1R8 was cloned, expressed and purified using E. coli host system. Turbidity assays, negative-stain electron microscopy (EM) and single-molecule fluorescence spectroscopy (SMFS) analysis indicated a potential interaction between IL-1R8 $8^{\mathrm{TIR}}$ and MAL ${ }^{\mathrm{TIR}}$. MAL ${ }^{\mathrm{TIR}}$ forms filamentous assemblies when incubated with IL-1R8 ${ }^{\mathrm{TIR}}$ (Fig. 1). We are currently focusing on solving the 3D structure of $\mathrm{MAL}^{\mathrm{TIR}} / \mathrm{IL}-1 \mathrm{R} 8^{\mathrm{TIR}}$ filaments using negative-stain EM and cryo-EM to obtain molecular insights into the interaction interfaces and binding sites of IL-1R $8^{\mathrm{TIR}}$ and MAL ${ }^{\mathrm{TIR}}$. This study will eventually lead to an understanding of how TLR4 signalling is regulated by IL-1R8 and can potentially pave way in development of new therapeutic agents in future.
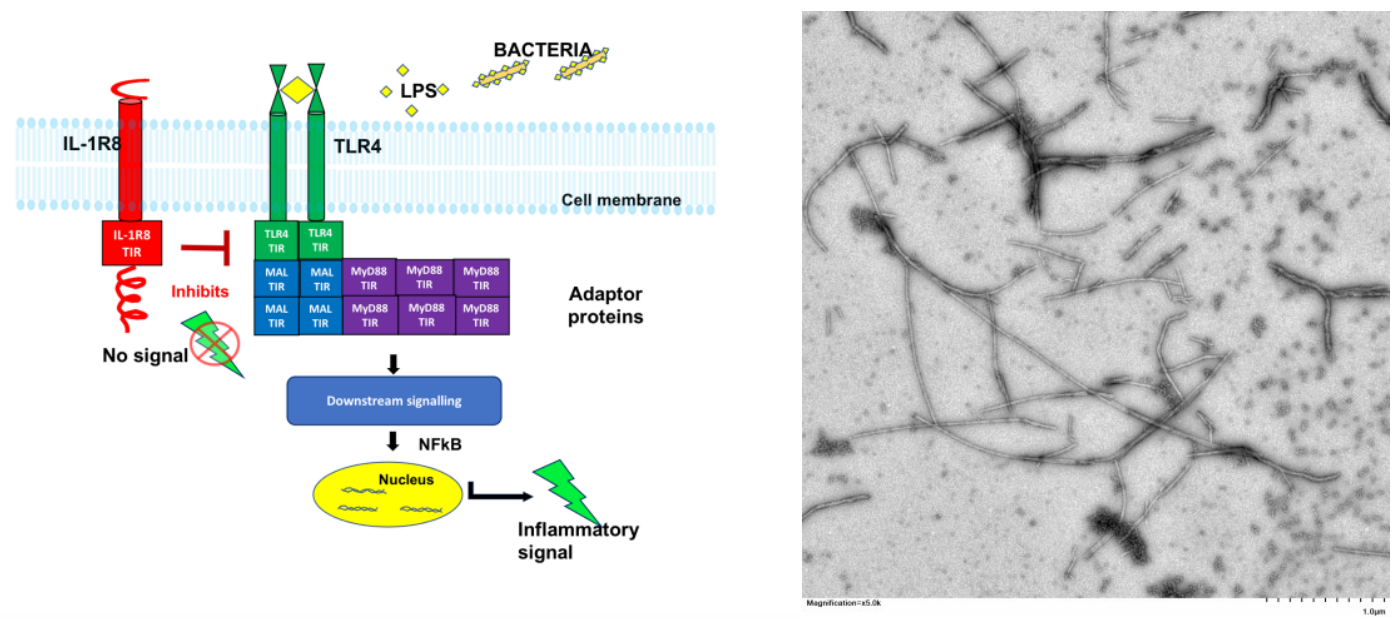

Figure 1. Left: Model representing the inhibition of TLR4 signalling by IL-1R8. Right: Negative-stain EM image of MALTIR/IL1R8TIR filaments taken using Hitachi HT 7700 TEM.

[1] Boraschi, D. et al. (2018). Immunol Rev. 281, 97-232

[2]. Qin, J. et al. (2005). J Biol Chem. 280, 25233-25241

[3]. Ve, T. et al. (2017). Nat. Struc. Mol. Biol. 24, 743-751

Keywords: Interleukin-1 receptor 8; TLR4 signalling; MAL/TIRAP; TIR domain; cryo-EM 